\title{
Avoiding anomalous newborns: preemptive abortion, treatment thresholds and the case of baby Messenger
}

Michael L Gross The University of Haifa, Haifa, Israel

\begin{abstract}
In its American context the case of baby Messenger, a preterm infant disconnected from life-support by his father and allowed to die has generated debate about neonatal treatment protocols. Limited by the legal and ethical norms of the United States, this case did not consider treatment protocols that might be available in other countries such as Denmark and Israel: threshold protocols whereby certain classes of newborns are not treated, and preemptive abortion allowing one to choose late-term abortion rather than risk delivery. Each offers a viable and ethically sound avenue for dealing with the economic and social expense of anomalous newborns by aborting or not treating those most likely to burden the health care system. Objections that these protocols are antithetical to American bioethical principles are considered but rejected as each policy answers to economic justice, utility and respect for autonomy.

(Fournal of Medical Ethics 2000;26:242-248)
\end{abstract}

Keywords: Abortion; selective non-treatment of newborns; comparative bioethics; neonatal care; neonaticide

\section{Introduction}

The case of baby Messenger, a severely ill preterm infant removed by his parents from ventilator support and allowed to die, serves to illustrate treatment options when infants are threatened by extremely premature birth. In most cases, alternative courses of action are defined within acceptable treatment protocols of impaired newborns. These include a "statistical" approach whereby treatment is withheld from infants defined as underweight and/or immature, an "initiate and reevaluate" approach whereby aggressive treatment is begun and then reevaluated relative to the infant's progress and parents' wishes, and a "treat until certainty" approach whereby each infant is treated until death or discharge.

Each of these approaches has attendant virtues and vices in the context of American neonatal care. But the Messenger case can also be used to examine prevailing norms and policies of different countries. Policymakers in Denmark, a country that accepts the statistical approach, view the ethical dimension of this case differently from those in the UK and the US where an "initiate and reevaluate" approach is accepted. Each of these treatment options remains, nevertheless, a neonatal protocol.
In each case, no decision is taken until after the baby is born. However, in the Israeli context, this case takes an entirely different turn. Israel's unrestrictive abortion policy may well permit the option of a late-term abortion thereby obviating any impaired infant protocol. The situation is exacerbated because neonatal policy in Israel conforms largely to the "wait until certainty" approach. In all instances, a closer look at the Messenger case illustrates the conundrum of abortion and neonatal care in a cross-national context.

\section{Saving baby Messenger}

Admitted for premature labour at twenty-five weeks gestation, Messenger was informed that her baby stood a $50-75 \%$ chance of mortality and a $20-40 \%$ chance of severe cerebral haemorrhage and neurological damage should he survive. The consulting neonatalogist also indicated a significant possibility of respiratory complications. With these statistics in mind, the parents instructed the attending physician not to undertake extraordinary efforts to save the life of the newborn. The neonatalogist, in turn, instructed her assistant to intubate the baby only if he was "vigorous" and "active". Although the baby weighed only 780 grams and was hypotonic and hypoxic these instructions were ignored, and the baby was resuscitated, intubated and incubated. The father, agonised that his instructions were not followed, removed his son from life-support, allowing him to die in his parents' arms.

This case, in which Messenger was charged with manslaughter and acquitted, generated a great deal of discussion regarding alternative avenues of neonatal care, parental rights, and the responsibility of the attending physician to honour parental requests to terminate care. By and large, the ethical discussion is limited by the legal options available in the United States where the case occurred, and is restricted therefore to the relative merits of the "initiate and reevaluate" and "wait until certainty" protocols. Reviewing the Messenger case, most commentators-considering both the infants's best interests and the need for implied or explicit consent-emphasise the need to consider parental interests while allowing parents greater say about the decision to terminate life-support..$^{2-5}$ However, two additional options deserve further exploration. First, a statistical approach stipulating thresholds below which young, immature infants would not be 
resuscitated merits review. Often dismissed by American commentators as fundamentally antithetical to American bioethics, this approach has been adopted by the Danish Council of Ethics. Second, there is room to discuss late-term abortion as a radically different solution to the Messenger case. Given the high mortality rate and significant possibility of impairment, would it not make sense to allow parents the option of abortion, thereby preempting the need for any other neonatal policy?

\section{Statistical non-treatment as a solution to the Messenger case}

In contrast to a strict statistical protocol that denies treatment to all members of a specific class of very extremely low birthweight (ELBW) infants, The Danish Council of Ethics endorses a modified threshold protocol that combines a minimum gestational age, a maturity criterion and respect for parental wishes. ${ }^{6}$ Under this protocol, infants younger than 24 or 25 weeks will not be aggressively treated. However, this threshold is modified by two conditions. First, mature infants, even those younger than 24 or 25 weeks may be revived if this can be accomplished using "low technology modalities" and minimal handling to induce respiration. $^{78}$

Second, the threshold is further modified by considerations of parental wishes. Viability is a function of the care a child can expect to receive from his parents, and is substantially impaired if the parents are unable or unwilling to provide the intensive care a preterm infant requires. As a result the threshold and maturity criterion may be overridden both by parents wishing to care for a child that fails to meet the criterion or by parents requesting to withhold treatment from a newborn that meets the threshold requirement. Under these guidelines, baby Messenger need not have been resuscitated. Even had the neonatalogist decided that the gestational threshold had been met, the baby's immediate condition following birth did not meet the maturity criterion. This, together with the parents refusal of ventilator support, should deter resuscitation. There is no real dilemma.

There are two distinct principles behind the council's recommendations - the infant's best interests and economic justice:

"The basis for the [modified threshold] recommendation is that the panel considers the $35 \%$ occurrence of severe handicaps in children born after a pregnancy term of 24-25 full weeks to be high in relation to the number of surviving infants; the panel also takes into account the comparison of the expenditure incurred with the possible alternative applications for that amount."

From this perspective sanctity of life is tempered by the infant's best interests and societal cost. On the one hand, a $35 \%$ risk of severe impairment is simply one that no parent, physician or policy-maker is prepared to inflict on a newborn. On the other, the possibility that the parents may bear the life-long emotional and financial cost of raising a handi- capped child is not offset by the likelihood that the child may none the less lead a relatively normal life. While this seems a reasonable decision for parents to make, it also means, by extension, that large numbers of healthy infants are sacrificed to avoid fewer numbers of handicapped infants. While 35 out of every 100 ELBW infants denied treatment would have been severely handicapped, the vast majority would have been normal or only moderately impaired. This outcome seems patently unjust unless tempered by other factors. Such a decision at the social level can only be justified if the attendant cost of neonatal care outweighs the lives of 65 relatively normal children (of every 100 denied treatment). This occurs if the money could be put to better use, ie saving more lives. The economic claim is pivotal. There is little interest in devoting resources either to lowering the $35 \%$ figure or to saving increasingly younger infants:

"It seems reasonable to exercise reticence in the treatment of extremely preterm infants in order to benefit the slightly less premature, since the prospects of better results increase with age and fewer resources are consumed, allowing more to be helped." 10

Given the high costs of neonatal care, economic considerations are difficult to assail. Unlike an individual parent's decision to withhold treatment from an ELBW infant, comprehensive policy to do so must be anchored in considerations of economic justice. This is a problem all public health systems grapple with as different groups vie for scarce resources. Interestingly, the council has compartmentalised the recipients of justice. The claims of ELBW newborns are not balanced against adults or other children, but against other premature infants. Under such a modified utilitarian plan one can presumably distribute resources equally between morally relevant groups (such as the elderly, premature, or terminally ill) while cost-benefit calculus can determine resource distribution within each group. This tempers the inherent defect of utilitarian justice that may medically impoverish certain groups in the interests of maximising overall utility.

\section{One obvious objection}

In this way, a threshold policy answers to the demands of economic justice, the (modified) demands of utility and some measure of equality as well. Parental discretion allays fears of heavyhanded paternalism while allowing determined parents to care for infants below the threshold or withhold treatment from those above it. Considerations of maturity partially resolve the problem of outliers, as some potentially viable infants are treated regardless of gestational age.

So what's wrong with a modified threshold policy? One obvious objection suggests that the statistical approach denies patients reasonable prognostic certainty. By denying all infants resuscitation the healthy are discarded with the handicapped. Would it not be more just and equally 
cost-effective if all infants were resuscitated and then evaluated with an eye towards terminating treatment for those most severely afflicted?

As attractive as this argument is, it easily undermines other treatment protocols as well. A typical American response might argue that failure to provide resuscitation is draconian, casting a net that denies a healthy life to viable infants as well. But there is no way to ensure that healthy infants won't die under any policy short of "treat until certainty". While resuscitation may offer the possibility that greater numbers of healthy infants survive, it ultimately falls to the same argument levelled at threshold protocols. Reevaluation and subsequent treatment decisions are based on probability figures no less than the decision to deny treatment at birth. The outcomes of high-grade haemorrhages, for example, might be equally indeterminate as those facing ELBW infants. Why not resuscitate and wait to evaluate the outcomes? But how far down the road should one reevaluate, two weeks, two months, two years? Is it then possible to discontinue treatment for a two- or three-year-old, while in the meantime creating a large pool of handicapped children? The argument based on relative prognostic certainty cannot work. It merely creates a slippery slope one might like to avoid. Resuscitation with the intent to reevaluate and, if necessary, discontinue treatment also blurs the distinction between withholding and withdrawing treatment. The oft-repeated claim that the two are ethically indistinguishable is not universally true. It certainly gives the Danish Council of Ethics pause for reflection while a hard distinction between the two suffuses Israeli case law and practice. Certainly the two are psychologically dissimilar. Parents asked to terminate life-support are not in the same position as those asked to acquiesce to withholding treatment. A threshold policy turns on this difference by making non-treatment the subject of presumed consent: unless parents choose otherwise no treatment is provided. Parental autonomy is affirmed but only covertly, in a manner similar to some European organ donation protocols. Ethically, presumed consent avoids what the council terms the "autonomy trap": the tendency to place too much responsibility on the shoulders of ill-prepared parents. Economically, this policy avoids the costs of extensive resuscitation. Only the most motivated and informed parents are apt to come forward, whether it be on the basis of a decision to care for a severely handicapped infant or to withhold treatment from an infant that might otherwise be treated. It is therefore not at all obvious that an "initiate and reevaluate" protocol affords similar cost-effectiveness as a threshold policy or is as ethically compelling.

\section{Presumed consent}

Finally it has been suggested that a threshold protocol smacks of sinister social planning that is antithetical to American individualism. ${ }^{5}$ But it is not immediately clear how this policy negates individualism. One might argue that the threshold approach does not really respect parental autonomy. But the force of this argument leans heavily on a rarified American vision of autonomy that is coming under increasing attack. ${ }^{11}$ In many ways, a modified threshold policy maintains respect for autonomy, albeit a gentler autonomy, largely by removing active decision making from the hands of the parents and relying on presumed consent. Additionally, one might argue that the statistical approach ignores the "ability of 'outliers' to survive or the willingness of some parents to cope with tragic circumstances". ${ }^{12}$ These are reasonable objections but they do not accurately reflect a modified policy tempered by considerations of maturity and parental discretion. Nor do they have much to do with individualism.

Individualism (or more specifically, "individuality") is, to cite Mill, inextricably linked to development and is best achieved when human beings are allowed to achieve the necessary moral, intellectual and practical development to become good citizens. ${ }^{13}$ The conditions necessary for continued human development form the basis of the modern welfare state. Oddly enough, American individualism denies its citizens basic health care, certainly one of the most necessary conditions for development. Does a threshold policy of neonatal care similarly deny one the conditions for development? On a very basic level the answer is yes, if it denies life to potentially healthy newborns. But this argument ultimately collapses into those already discussed and discarded. First, any treatment protocol that allows termination of life-support prior to absolute certainty will deny some the chance of a normal life. Second, health care systems are designed not only to provide life for the greatest number but also quality of life for the greatest number. There is no reason to assume that threshold protocols curtail medical services so crucial for personal development. On the contrary, a threshold policy enhances the availability of funds for other health care needs by eliminating an expense that benefits relatively few children.

Finally, one may object that threshold protocols return us to the ever-present slippery slope: if we refuse to treat a large class of newborns, what will prohibit us from aborting late-term fetuses whose odds of survival are similar to those of baby Messenger? What indeed?

\section{Preemptive abortion as a solution to the Messenger case}

Following US jurisprudence, American commentators often define abortion as the "expulsion of an embryo or fetus before it is viable". ${ }^{14}$ This makes it difficult to speak of "late-term" abortions, for in the American sense these are not abortions at all, but acts of feticide. From this perspective it is therefore necessary to distinguish between abortion as the termination of a pregnancy and abortion as the termination of a fetus. The former is a constitutionally protected right in the US, while the latter is justified only under rare conditions of fetal best interest. ${ }^{15}$ 
Abortion in Israel, on the other hand, is broadly defined as termination of pregnancy without regard to gestational age or fetal viability and, at later stages, inevitably includes fetal termination. Abortion at all stages of pregnancy is freely available for any of the following conditions: maternal age $(<17$ or $>40$ years of age), premarital pregnancy (or pregnancy resulting from rape or incest), danger to the mother's physical or mental health and/or fetal birth defects. Requests for abortion must be approved by a hospital committee. Abortion law is similar in Denmark while provisions in the UK specify "severe" fetal anomalies. In spite of the flexibility of the law, late-term (third trimester) abortions are generally avoided in the UK and Denmark. Israel, on the other hand, has one of the highest rates of late-term abortion in the world. ${ }^{16}$

The purpose here is not to explore the merits of late-term abortion policy (as discussed elsewhere ${ }^{16}$ ) but to reflect on the feasibility of preemptive abortion: terminating a normal fetus similar to the Messengers' in order to avoid dealing with the possibility of a severely malformed newborn. The issue is brought in the Israeli context, not because preemptive abortion is practised, but because it has been raised as an ethically problematic possibility that might be allowed under current abortion regulations. Moreover, neonatal treatment protocols in Israel reflect the "treat until certainty" approach. In the event of spontaneous delivery, any live, viable newborn would be treated aggressively. As a result preemptive abortion is the only way to avoid a malformed newborn when faced with severely premature birth. Under this scenario a woman in Messenger's position would be allowed the option of fetal termination when it was determined that she faced the probability of delivering an anomalous infant. The immediate justification for a late-term abortion focuses on the high probability of severe deformity facing the infant. The immediate objection, on the other hand, focuses on the health of the fetus: in utero s/he is completely normal.

The justification for abortion unpacks to include the same kind of probability calculations that inform a threshold or "initiate and reevaluate" protocol. If one accepts a $35 \%$ chance of severe disability as a reasonable threshold to withhold treatment, or the Messenger statistics as a reasonable threshold to withdraw treatment, then why not accept these same probabilities to justify a late-term abortion? What is the difference between a late-term fetus and an early-term infant? If the answer is none then the two must be treated equally. One will not arbitrarily abort fetuses with minor anomalies anymore than one will discontinue treatment for minimally ill newborns nor will one save either with major anomalies. Regardless of the difficulties of defining minor or major anomalies, there is little doubt that the Messenger case represents a high probability of a severely handicapped child. An anomaly that is grounds for withholding life-support must also be grounds for aborting the same fetus.
Absent any crucial difference between the fetuses and newborns any reluctance not to treat the two cases similarly is difficult to understand. Nevertheless, many nations, Denmark, the United States and Great Britain for example, permit one to terminate life-support in the case of anomalous newborns but are reluctant to abort a fetus (in this case the very same fetus) facing similar probabilities of impairment. There is no room for the decision making process that emerges from most analyses of the Messenger case: a carefully balanced consideration of the baby's best interests and deference to parents' desires based on the interests and costs of those who must raise the child.

\section{Subtle shift}

The sources of this confusion are not clear. It may reflect a misguided quest for reasonable prognostic certainty on the assumption that birth itself may afford some better prognostic perspective or it may reflect influential religious norms. On the latter view, fetal life is sacrosanct while selective non-treatment of newborns is subsumed under the general, permissible practice of withholding or withdrawing medical treatment from any patient. Notice, however, how this reasoning produces a subtle shift in fetal/newborn status. Late-term fetuses and preterm infants no longer enjoy similar status. Instead, the former is elevated with respect to the latter, giving fetuses greater protection than newborns. Similarly, the US Supreme Court protects fetal life to a degree to which it does not protect the neonate. Without this move it is impossible to justify any cogent opposition to a threshold policy or preemptive abortion in those cases of fetuses or newborns facing similar odds of survival.

As it happens the case is somewhat easier to resolve in Israel. There, the status of the late-term fetus and newborn are not identical, for legal and moral personhood are only conferred at birth. Prior to birth, the mother's discretion is paramount. Nevertheless, Israeli law will not allow capricious abortion: the fetus must be malformed, even if the effects of the anomaly are difficult to determine. But in this case the fetus is not malformed. Is there then any ethical relevance to the fact that the fetus in utero is normal, the proximate cause of his anomaly only his impending birth? This is not only an Israeli dilemma. The dilemma also arises in those cultures which view the status of the late-term fetus and the newborn as identical. Any difference in treatment between prenatal and neonatal baby Messenger must reflect some fundamental difference between the two. Can this be found in the distinction between an observed anomaly and an impending anomaly?

Although each anomaly is qualitatively distinct it is currently technologically impossible to separate the fetus's condition in utero from those of his or her imminent birth. Were this possible, alternative justifications would have to be provided for terminating a late-term healthy fetus. One attempt to do so, based on "our duty to avoid bringing 
unwanted children into a community that is not prepared to accommodate them" has met with considerable resistance. ${ }^{17}{ }^{18}$ Absent this technology, the Messenger baby is absolutely certain to face the odds cited by the consulting neonatalogist: an unavoidable, impending anomaly is extensionally equivalent to an observed anomaly.

Decision making is now relatively straightforward. If fetal and newborn status is similar, the factors informing the decision to abort are identical to those informing termination of neonatal lifesupport: right to life, quality of life and utility. On the one hand, fetal right to life and reasonable medical care are subject to the same considerations of futility and/or low cost-benefit ratio as that of a newborn. In spite of fetal rights and personhood, we should permit preemptive abortion under the same conditions that we may discontinue lifesupport. On the other hand, if withholding or withdrawing life-support can be justified by considerations of the infant's and/or family's best interests, so must preemptive abortion.

If the newborn and fetal status are not similar one can still reason from intuitively sound cases of observed fetal anomaly that justify late-term abortion. Severe spina bifida or hydrocephalus are conditions with a high probability of poor outcomes. A decision to abort such a fetus in the third trimester reflects legitimate considerations of fetal and parental best interests. ${ }^{19}$ Absent any significant difference between the probable outcomes of these observed anomalies and the threat posed by extremely premature birth, preemptive abortion is justified.

\section{Active euthanasia}

If the latent nature of the anomaly fails to repudiate preemptive abortion, perhaps the difference lies in the form of abortion. In Israel, live abortuses are precluded as late-term abortion is accomplished by intracardial injection of potassium chloride. If the status of the fetus and the newborn are similar, isn't this similar to the lethal injection of a newborn? Condemning the latter also condemns the former. One cannot abort baby Messenger because it moves us unjustifiably close to active euthanasia.

To work through this objection it is important to understand why one might want to terminate a fetus. Considerations of a mother's health may not be a relevant factor, for this usually only justifies termination of pregnancy. Fetal death is a separate act that must be justified in its own right. Most often, justification for fetal termination is anchored in fetal best interests. This can occur in two cases. First, fetal termination is justifiable when the fetus is afflicted with a disease, such as Tay-Sachs, from which it will certainly die. Second, it is justified when a fetus suffers from an affliction, such as Lesch Nynan syndrome, that makes life worse than death. Because these anomalies can often be diagnosed in the second trimester, late-term abortion is relatively rare. Nevertheless, it is important to notice how these justifications for fetal termination place the fetus in a different category from the newborn. To terminate similarly afflicted newborns by lethal injection is nothing short of murder in most nations. While one could withhold or withdraw treatment, any form of active euthanasia is prohibited. As a result of these considerations it is apparent that the status of the late-term fetus is inferior to that of the preterm newborn. There are, therefore, cases that justify fetal termination by lethal injection. Would they extend to the Messenger case?

Once fetal personhood is discounted to allow for the use of lethal injection in cases of certain death or severe malformation accompanied by pain and suffering, the door cracks open for other considerations as well. While in each of these cases, death or suffering is certain, the decision ultimately reflects the parent's assessment of the infant's best interests. This occurs in innumerable other instances as well, where one encounters a high probability of certain death or intense suffering. Parents diagnosed with Tay-Sachs carrier status in the days before fetal screening might legitimately opt for late-term abortion. So might a parent facing the birth of a child with neural tube defects whose probabilities of morbidity are no better than those facing the Messengers. Assuming no significant distinction between an observed and impending anomaly, there is no consistent way to deny preemptive abortion to parents facing the Messenger dilemma unless one absolutely prohibits the use of fatal fetal injection based on the general prohibition of its use among persons, while at the same time reasserting fetus/newborn parity. ${ }^{20} 21$

Preemptive abortion may, nevertheless, fly in the face of deeply held intuitions about the sanctity of life and the natural repugnance associated with lethal injection. Would it not be easier to allow nature to take its course and simply allow spontaneous labour to culminate in delivery thereby avoiding the intentional killing of a fetus, whatever its status? This might be true were spontaneous delivery the only decision to be taken by parents or physicians. The fact remains, however, that soon after delivery one must still decide to withhold, terminate or continue some form of treatment. As suggested above, these options can be equally problematic. If, as in the Israeli case, personhood is attained only at a child's birth then anything less than aggressive treatment may be as repugnant as lethal injection. Moreover, it must be remembered that a conservative "initiate and reevaluate" management strategy confers no better vantage point from which to predict a patient's outcome; prognostic certainty remains equally elusive at any stage in the treatment of malformed children. Finally, preemptive abortion and threshold protocols largely allay the anguish of bearing and raising a severely impaired child. These considerations should not be discounted. 


\section{Concluding comments}

When examined in an international, cross-cultural context, bioethical dilemmas yield alternative policy directions that are not always apparent in their original context. There is no easy way to face the possibility of anomalous newborns, but might not the alternatives analyzed here, preemptive abortion and threshold protocols, provide feasible policy options as bioethics evolves in those countries discussed above?

In Denmark, thresholds are largely justified by the economic imperative to ration scarce medical resources and the ethical imperative to alleviate parental distress while safeguarding parental autonomy through presumed consent. For the time being the two go hand in hand, but one can easily imagine a situation where some technological advance would allow one to guarantee the health of a premature infant, thereby alleviating emotional costs but exacerbating economic expense. At this point it appears that budgetary considerations are paramount:

"... the [threshold] approach has the strength of not stimulating endeavors to become increasingly better at saving increasingly young fetuses ... . It is thus instrumental - indirectly, at least - in maintaining a level of skepticism about techno-crazed fantasies of engineering 'the artificial womb'."10

In spite of this admonition, there is some evidence to suggest that Danish physicians may be moving away from a modified threshold approach towards an "initiate and reevaluate" strategy for managing ELBW infants. ${ }^{22}$ While this trend remains to be confirmed Norup suggests that it may partially be explained by some of the factors whose importance the Council of Ethics, ironically, sought to dispel: increased survival rates of ever smaller infants and a blurring of the distinction between withdrawing and withholding treatment, thereby making it increasing palatable to begin and later withdraw treatment.

In Israel on the other hand, preemptive abortion would most likely be only a stopgap measure. Given the current bioethical climate in Israel where fertility and aggressive treatment are both overriding norms, Israelis would probably opt for any measure, including an artificial womb, that would let parents avoid a late-term abortion while insuring a normal newborn. Preemptive abortion is not a budgetary imperative but a treatment option to relieve parents of the anguish of dealing with an anomalous newborn. If other measures are available, so much the better. Nevertheless, the Danish Council of Ethics raises an issue that cannot be ignored: when is enough enough insofar as neonatal care is concerned?

Recent research has not produced unequivocal answers. Some suggest, for example, that resuscitation followed by evaluation postpones median time to death by only two days. ${ }^{23}$ However, resuscitation also saved $20 \%$ of the 23- and 24-week-old newborns in this particular sample, newborns that most likely would not have been resuscitated under
Danish guidelines and possibly aborted under current Israeli regulations. Although neonatalogists continue to argue that "medical resources allocated to non-survivors remain low", ${ }^{24}$ this misses the point that threshold protocols and preemptive abortion are driven by the material and emotional costs associated with raising the surviving anomalous newborn.

Policy decisions ultimately come down to ethics and economics. In spite of the possibility of improved neonatal technology the Danish council prefers not to invest in this direction, leaving resources to serve a greater number of citizens. Israelis, on the other hand, may be willing to wait for advanced technology to ensure greater fertility rates and alleviate the distress caused by aborting a normal late-term fetus. The American dilemma is more pronounced. They have no special interest in population growth, nor are they oblivious to rising heath care costs. While they should be prime candidates for threshold protocols or preemptive abortion, both options are largely proscribed by state and federal law. Nevertheless, one of ethic's prime functions is to counsel the law and if the arguments supporting each of these two policies are persuasive then there are good reasons for placing these issues on the public agenda.

Michael L Gross, PhD, is Senior Lecturer in the Department of Political Science,The University of Haifa, Haifa, Israel.

\section{References and notes}

1 Paris JJ. Parental right to determine whether to use aggressive treatment for an early gestational age infant: the Messenger case. Medicine and Law 1997;16:679-85.

2 Clark FI. Making sense of state vs Messenger. Pediatrics 1996; 97,4:579-83

3 Messenger G. No Sense making sense of state vs Messenger. Pediatrics 1997;99,2:306.

4 Harrison H. Commentary: the Messenger case. fournal of Perinatalogy 1996;16,4:299-301.

5 Paris JJ, Schreiber MD. Parental discretion in refusal of treatment for newborns: a real but limited right. Clinics in Perinatalogy 1996;23,3:573-81.

6 Danish Council of Ethics. Debate outline: extreme prematurity, ethical aspects. Copenhagen: Eurolingua, 1995: 28-9.

7 See reference 6:19-21

8 Jakobson T, Gronvall J, Petersen S, Andersen GE. "Minitouch" treatment of very-low-birthweight infants. Acta Paediatrica treatment of ver

9 See reference $6: 28$

10 See reference 6: 36

11 Gross ML. Autonomy and paternalism in communitarian society: patient rights in Israel. Hastings Center Report 1999;29,4:13-20

12 See reference 5: 577

13 Mill JS. On liberty. In: Collini S, ed. On liberty and other writings. Cambridge: Cambridge University Press, 1989: ch 3: 56.

14 The American heritage Stedman's medical dictionary. Boston: Houghten Mifflin, 1995:3

15 Mahowald MB. Concepts of abortion and their relevance to the abortion debate. Southern fournal of Philosophy. 1982; Summer: 195-207.

16 Gross ML. After feticide: coping with late-term abortion in Israel, Western Europe and the United States. Cambridge Quarterly of Healthcare Ethics 1999;8,4:449-62.

17 Callahan J. Ensuring a stillborn: the ethics of fetal lethal injection in late abortion. Fournal of Clinical Ethics 1995;6,3:254-63.

18 Fletcher JC. On learning from mistakes. fournal of Clinical Ethics $1995 ; 6,3: 264-70$ 
19 Abortion of late term fetuses with a high probability of severe anomaly is not without controversy. However, resistance is highest in those nations that grant fetal/newborn parity. See reference 16.

20 Isada NB, Pryde PG, Johnson MP, Hallak M, Blessed WB Evans MI. Fetal intracardiac potassium chloride injection to avoid the hopeless resuscitation of an abnormal abortus: I clinical issues. Obstetrics and Gynecology 1992;80:296-9.

21 See references 17 and 18 and also Fletcher JC, Isada NB, Pryde PG, Johnson MP, Evans MI. Fetal intracardiac potassium chloride injection to avoid the hopeless resuscitation of an ride 1992:80:310-13. Theser. late, second trimester pregnancies. While Fletcher, for example, supports using fetal injection in second trimester pregnancies he is reluctant to support third trimester abortions. Callahan, however, is less restrained.

22 Norup M.Treatment of extremely premature infants: a survey of attititudes among Danish Physicians. Acta Paediatrica 1998; 87:896-902.

23 Doron MW, Veness-Meehan KA, Margolis LH, Holoman EM, Stiles AD. Delivery room resuscitation decisions for extremely premature infants. Pediatrics 1998;102:574-82.

24 Meadow W, Reimshisel T, Lantos J. Birth weight-specific mortality for extremely low birth weight infants vanishes by four days: epidemiology and ethics in the neonatal intensive care unit. Pediatrics 1996;97:636-43.

\section{Editor}

\section{Journal of Medical Ethics}

Applications are invited for the editorship of the Journal of Medical Ethics. This position will fall vacant from April 2001 on the retirement of Professor Raanan Gillon. The journal is jointly owned and published by the Institute of Medical Ethics and the BMJ Publishing Group.

The successful candidate(s) is likely to have academic qualifications and proven expertise in teaching and/or writing in the field of medical ethics. While neither previous editorial experience nor medical qualifications are strict requirements, either or both will be an advantage. Joint applications from two candidates wishing to act as co-editors may be considered.

The successful applicant(s) will be required to maintain close links with the Institute. A threemonth probationary period is envisaged.

Please send a letter of application, a curriculum vitae, a statement of the strengths and weaknesses of the journal and brief resume of your proposed editorial policy. The journal is currently a bimonthly publication but a change to monthly publication is being considered.

Closing date for applications is 30th September 2000 and interviews will be held during October to enable the successful candidate to take up the post in 2001.

Further details of the post are available from Alex Williamson at the address below. Raanan Gillon is also available to give advice.

Mrs Alex Williamson, BMJ Publishing Group, BMA House, Tavistock Square, London WC1H 9JR, UK.

Telephone: $+44(0) 2073836169$;

Fax: +44 (0)20 7383 6668;

email: awilliamson@bmjgroup.com r.gillon@ic.ac.uk 